\title{
Clinical Outcomes of Mixed Response to Pembrolizumab in Advanced Urothelial Carcinoma After Platinum-based Chemotherapy
}

\author{
NOBUKI FURUBAYASHI ${ }^{1}$, TAKAHITO NEGISHI ${ }^{1}$, NAOTAKA SAKAMOTO ${ }^{2}$, SHINGO TAMURA ${ }^{3}$, \\ FUTOSHI MOROKUMA ${ }^{4}$, YOOHYUN SONG ${ }^{5}$, YOSHIFUMI HORI ${ }^{6}$, TOSHIHISA TOMODA ${ }^{7}$, \\ NARIHITO SEKI ${ }^{5}$, KENTARO KUROIWA ${ }^{6}$ and MOTONOBU NAKAMURA ${ }^{1}$ \\ ${ }^{1}$ Department of Urology, National Hospital Organization Kyushu Cancer Center, Fukuoka, Japan; \\ ${ }^{2}$ Department of Urology, National Hospital Organization Kyushu Medical Center, Fukuoka, Japan; \\ ${ }^{3}$ Department of Medical Oncology, National Hospital Organization Kyushu Medical Center, Fukuoka, Japan; \\ ${ }^{4}$ Department of Urology, Saga-ken Medical Centre Koseikan, Saga, Japan; \\ ${ }^{5}$ Department of Urology, Kyushu Central Hospital of the Mutual Aid Association \\ of Public School Teachers, Fukuoka, Japan; \\ ${ }^{6}$ Department of Urology, Miyazaki Prefectural Miyazaki Hospital, Miyazaki, Japan; \\ ${ }^{7}$ Department of Urology, Oita Prefectural Hospital, Oita, Japan
}

\begin{abstract}
Background/Aim: Despite the presence of a mixed response $(M R)$ in patients with urothelial carcinoma (UC) who receive immune checkpoint inhibitors, the clinical outcome of these patient has not been reported. We evaluated the clinical outcome of MR to pembrolizumab for advanced UC. Patients and Methods: Advanced UC patients who received pembrolizumab after platinum-based chemotherapy failure with measurable disease in multiple organs were retrospectively analyzed. Results: Among 31 patients, MR [including progressive disease $(P D)+$ complete response $(C R)$ or partial response (PR)] was confirmed in 4 (12.9\%). The median overall survival (OS) of the $C R+P R$ (including $C R+S D \pm P R$ ), stable disease $(S D), P D$ (including $P D \pm S D$ ) and MR groups was 16.0, 5.1, 5.4 and 4.3 months, respectively. There was no significant difference in the $O S$ between the MR and CR+PR response groups (log-rank test, $p=0.069$ ). Conclusion: A mixed response to pembrolizumab in advanced UC was not uncommon. Despite the nonsignificant difference in the OS between the mixed and
\end{abstract}

This article is freely accessible online.

Correspondence to: Nobuki Furubayashi, Department of Urology, National Hospital Organization Kyushu Cancer Center, Notame 31-1, Minami-ku Fukuoka 811-1395, Japan. Tel: +81 925413231, Fax: +81 925514585, e-mail: nobumduro@gmail.com

Key Words: Urothelial carcinoma, pembrolizumab, mixed response, organ-specific response, tumor microenvironment.
$C R+P R$ response groups, the $O S$ of the $M R$ group tended to be similar to that of the $S D$ and $P D$ response groups.

The advent of immune checkpoint inhibitors (ICIs) has revolutionized the therapeutic landscape for multiple malignancies, including advanced urothelial carcinoma (UC), resulting in significant improvements in patients' survival. $\mathrm{UC}$ is characterized by genomic instability, high expression of the programmed cell death ligand 1 (PD-L1) protein, DNA damage-response mutations and a high tumor mutational burden, all of which are associated with an increased response to ICIs in several cancer types $(1,2)$.

At present, in Japan, pembrolizumab (anti-PD-1 antibody) and avelumab (anti-PD-L1 antibody) have been approved for advanced UC. Pembrolizumab is associated with a significantly longer overall survival (OS) and a lower rate of treatment-related adverse events compared to chemotherapy as second-line therapy for platinum-refractory advanced UC (3). Avelumab was recently approved because using it in maintenance therapy in combination with excellent supportive care of patients and comparing it to the effect of the same supportive care alone, showed that the first significantly prolonged the OS of patients with UC, who had disease that had not progressed after first-line chemotherapy (4).

Despite these improvements, however, the evaluation of individual patient response to immunotherapy can be complex and unpredictable. Compared to conventional chemotherapy and molecular-targeted drug therapy, ICIs can induce novel therapeutic responses, such as pseudoprogression, which 
involves an initial increase in the size of the tumor lesion with subsequent tumor shrinkage and hyperprogresion, which is characterized by accelerated disease progression and a reduced survival duration (5-10). In addition, a given individual can have at the same time regression in some tumors and progression in others, a phenomenon termed as a mixed response (11). ICIs induce antitumor effects by reactivating exhausted $\mathrm{T}$ cells and thus rejuvenating the antitumor immunity. Therefore, the differential tumor microenvironments of the various organs may influence the therapeutic effect of ICIs (12), and, thus, ICIs may also be able to induce a mixed response.

In the present study, we retrospectively assessed the clinical outcomes of a mixed response to pembrolizumab in patients that failed to respond to platinum-based chemotherapy for advanced UC.

\section{Patients and Methods}

The present study was approved by the Institutional Review Board of National Hospital Organization Kyushu Cancer Center (2020-28), and a written informed consent was obtained from all patients.

Definition of a mixed response. 'Mixed response' was defined as a measurable disease in multiple organs, including the primary tumor organ. The characteristics of the mixed response include a worsening response leading to progressive disease (PD) and an improved response leading to complete response (CR) or partial response (PR) in the same patient.

Patient population. We identified 81 consecutive patients with advanced UC who received pembrolizumab following failure of platinum-based chemotherapy at 6 institutions between January 2018 and October 2020. All patients were histopathologically diagnosed with UC and showed a radiologically confirmed disease progression after platinum-based chemotherapy (13). Only patients who had measurable disease in multiple organs (not just a single organ), including the primary tumor organ, and for whom subsequent imaging findings were available for the evaluation of the response were enrolled. Ultimately, 50 patients were excluded from this study; 12 due to unmeasurable disease or a lack of subsequent imaging findings and 38 due to single-organ metastatic disease. Clinical data were retrieved from the patients' medical records.

The present study protocol was approved by the ethics committee of each institution and complied with the 1964 Declaration of Helsinki and its later amendments.

The response evaluation. Tumor measurements were generally performed using computed tomography before and after every four to six cycles of pembrolizumab; however, evaluations were performed as needed when the clinical symptoms worsened.

All metastases that measured $\geq 5 \mathrm{~mm}$ in the long axis [lymph node (LN) metastases $\geq 10 \mathrm{~mm}$ in the short axis] in computed tomography images were defined as measurable lesions and assessed before and during pembrolizumab treatment. For each organ-specific response, the tumor burden was defined as the sum of the long axis for all non-LN metastases or the short axis of all
Table I. Patient characteristics.

Characteristics $(\mathrm{n}=31)$

Age (years), median (IQR)

$73(61-78)$

Male gender, n (\%)

$21(67.7)$

ECOG PS score, n (\%)

0

$\geq 1$

$16(51.6)$

$15(48.4)$

Primary tumor site, $\mathrm{n}(\%)$

Upper urinary tract

$16(51.6)$

Bladder

$7(22.6)$

Upper urinary tract+bladder

$8(25.8)$

Pure UC in histologic testing, $\mathrm{n}(\%)$

$28(90.3)$

Number of chemotherapy regimens

before pembrolizumab, $\mathrm{n}(\%)$

2

3

$22(70.9)$

$3(9.7)$

$6(19.4)$

$\mathrm{Hb}<10 \mathrm{~g} / \mathrm{dl}, \mathrm{n}(\%) \quad 14(45.2)$

$<3$ months since previous chemotherapy, n (\%) 27 (87.1)

Number of diseased organs

(including primary tumor organ)

$15(48.4)$

$9(29.0)$

$7(22.6)$

4

Disease site, $\mathrm{n}(\%)$

Lymph node $20(64.5)$

Lung 19 (61.3)

Primary tumor organ (pelvis, ureter and bladder) $14(45.2)$

Liver

$9(29.0)$

Local recurrence $\quad 9(29.0)$

Bone $6(19.4)$

Subcutaneous $2(6.5)$

Muscle $2(6.5)$

Others (one organ each) $4(12.9)$

IQR: Interquartile range; ECOG PS: Eastern Cooperative Oncology Group Performance Status; UC: urothelial carcinoma; Hb: Hemoglobin.

LN metastases that were measured and evaluated according to the Response Evaluation Criteria in Solid Tumors (RECIST), version 1.1 (14).

For each metastatic organ, including with the primary tumor, the best response was classified as: i) CR (disappearance or reduction to $<10 \mathrm{~mm}$ in the short axis for all $\mathrm{LN}$ metastases), ii) PR ( $>30 \%$ reduction), iii) stable disease (SD) (neither CR, PR nor PD), and iv) PD (>20\% growth) (15-17). In addition, a mixed response was judged to be positive when measurable disease in multiple organs showed differing responses (e.g., PD plus either CR or PR in the same patient).

Statistical analyses. All statistical analyses were performed using the JMP ${ }^{\circledR}$ Pro, version 15.1.0 software package (SAS Institute, Inc., Cary, NC, USA). The Kaplan-Meier method was used to evaluate the OS, which was calculated from the day on which pembrolizumab was started until the date of the last follow-up examination or death from any cause, and the difference among the response groups was determined using the log-rank test. Statistical significance was set at $p$-Value $<0.05$. 
Table II. Objective response rate in patients treated with pembrolizumab.

\begin{tabular}{lc}
\hline Response $(\mathrm{n}=31)$ & Number of patients \\
\hline $\mathrm{CR}+\mathrm{PR}+\mathrm{SD}$ & 1 \\
$\mathrm{CR}+\mathrm{SD}$ & 2 \\
$\mathrm{SD}$ & 4 \\
$\mathrm{PD}+\mathrm{SD}$ & 13 \\
$\mathrm{PD}$ & 7 \\
$\mathrm{PD}+\mathrm{CR}+\mathrm{PR}$ & 1 \\
$\mathrm{PD}+\mathrm{PR}$ & 2 \\
$\mathrm{PD}+\mathrm{CR}+\mathrm{SD}$ & 1 \\
\hline
\end{tabular}

CR: Complete response; PR: partial response; SD: stable disease; PD: progressive disease.

\section{Results}

Patient characteristics. The clinical characteristics of the 31 patients [male, $\mathrm{n}=21$; female, $\mathrm{n}=10$; median age $=73$ years old; interquartile range $(\mathrm{IQR})=61-78$ years old] are listed in Table I. The median follow-up period was 5.4 months. All patients received pembrolizumab for UC after the failure of platinum-based chemotherapy. According to the Eastern Cooperative Oncology Group Performance Status (ECOG PS), $16(51.6 \%)$ and $15(48.4 \%)$ patients had a PS of 0 and $\geq 1$, respectively. Sixteen patients had upper urinary tract UC (51.6\%), 7 had bladder UC (22.6\%), and 8 had both types $(25.8 \%)$. The number of treatments attempted before pembrolizumab was as follows: i) 1 treatment $(n=22$, $70.9 \%)$, ii) 2 treatments $(n=3,9.7 \%)$ and iii) 3 treatments $(\mathrm{n}=6,19.4 \%)$. In the majority of patients, a histological examination revealed pure UC $(90.3 \%)$, and the time from the previous chemotherapy was $<3$ months $(87.1 \%)$. Before the start of treatment with pembrolizumab, the number of diseased organs, including the primary tumor organ, was 2 organs in 15 patients $(48.4 \%), 3$ organs in 9 patients and 4 organs in 7 patients $(22.6 \%)$. Disease target lesions were found at the following sites: i) lung $(64.5 \%, n=20)$, ii) lymph nodes $(61.3 \%, \mathrm{n}=19)$, iii) primary tumor organ (pelvis, ureter and bladder, $45.2 \%, \mathrm{n}=14)$, iv) liver $(29.0 \%, \mathrm{n}=9)$, v) local recurrence $(29.0 \%, \mathrm{n}=9)$, vi) bone $(19.4 \%, \mathrm{n}=6)$, vii) subcutaneous $(6.5 \%, \mathrm{n}=2)$, muscle $(6.5 \%, \mathrm{n}=2)$ and viii) others $(12.9 \%, \mathrm{n}=4)$.

Objective response rate and modified objective response rate in patients treated with pembrolizumab. Among the 31 patients who received pembrolizumab, the same response within multiple target organs was confirmed in 11 patients $(35.5 \%)$, with SD in 4 patients and PD in 7 patients. The remaining 20 patients $(64.5 \%)$ had different responses in separate target organs, including $\mathrm{CR}+\mathrm{SD} \pm \mathrm{PR}$ in 3 patients, $\mathrm{PD}+\mathrm{SD} \pm \mathrm{CR}$ in 14 patients and $\mathrm{PD}+\mathrm{PR} \pm \mathrm{CR}$ in 3 patients (Table II).
Table III. Modified objective response rate in patients treated with pembrolizumab.

\begin{tabular}{lc}
\hline Modified response $(\mathrm{n}=31)$ & Number of patients $(\%)$ \\
\hline $\mathrm{CR}+\mathrm{PR}$ & $3(9.7)$ \\
$\mathrm{SD}$ & $4(12.9)$ \\
$\mathrm{PD}$ & $20(64.5)$ \\
Mixed response (PD+CR or PR) & $4(12.9)$
\end{tabular}

CR: Complete response; PR: partial response; SD: stable disease; PD: progressive disease.

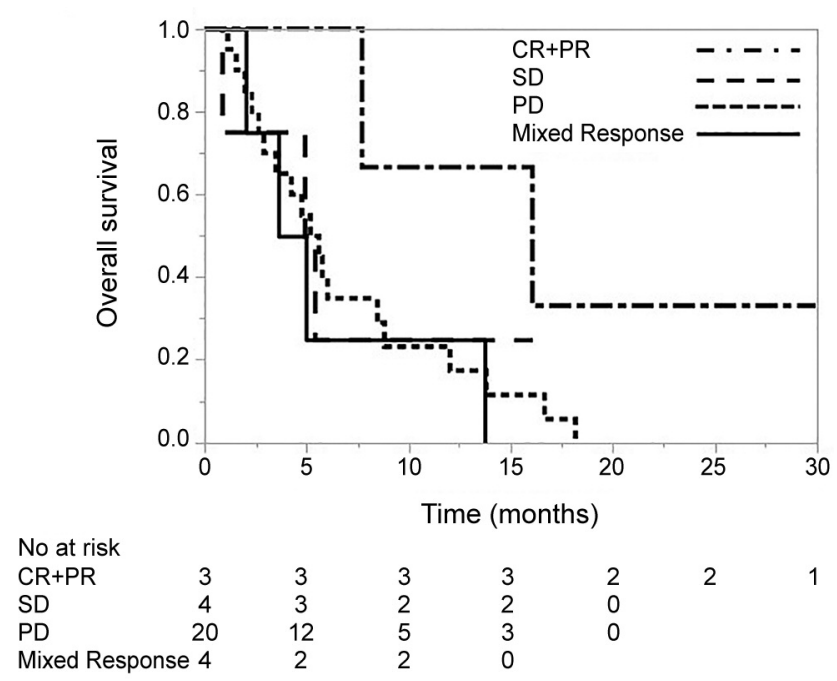

Figure 1. The overall survival in patients treated with pembrolizumab according to the modified objective response rate. CR: Complete response; $P R$ : partial response; $S D$ : stable disease; PD: progressive disease.

After reorganizing according to the modified objective response rate, i) $\mathrm{CR}+\mathrm{PR}$ (including $\mathrm{CR}+\mathrm{SD} \pm \mathrm{PR}$ ) was confirmed in 3 patients $(9.7 \%)$, ii) SD in 4 patients $(12.9 \%)$, iii) $\mathrm{PD}$ (including $\mathrm{PD} \pm \mathrm{SD}$ ) in 20 patients $(64.5 \%)$ and iv) mixed response (including $\mathrm{PD}+\mathrm{CR}$ or $\mathrm{PR}$ ) in 4 patients (12.9\%) (Table III).

The OS of patients treated with pembrolizumab according to the modified objective response rate. The OS according to the modified objective response rate for pembrolizumab is shown in Figure 1. The median OS in the CR+PR, SD, PD and mixed response groups was 16.0 months [95\% confidence interval $(\mathrm{CI})=7.6$ to not estimable], 5.1 months $(95 \% \mathrm{CI}=0.8$ to not estimable), 5.4 months (95\% CI=2.6 to 8.4) and 4.3 months (95\% CI=2.0 to 13.7), respectively. A log-rank test revealed no significant differences in the OS between the mixed response and the $\mathrm{CR}+\mathrm{PR}$ groups ( $p=0.069$ ), or in comparison to the SD $(p=0.595)$ and $\mathrm{PD}$ response groups $(p=0.702)$. 


\section{Discussion}

In this retrospective study, we analyzed the response of advanced UC patients (according to RECIST 1.1 criteria) to the ICI pembrolizumab after the failure of platinum-based chemotherapy. We categorized the response to therapy as: i) modified $\mathrm{CR}+\mathrm{PR}(\mathrm{CR}+\mathrm{SD} \pm \mathrm{PR})$, ii) $\mathrm{SD}$, iii) $\mathrm{PD}(\mathrm{PD} \pm \mathrm{SD})$ and iv) mixed (PD plus $\mathrm{CR}$ or PR), depending on the behavior of the tumor in the metastatic or primary organs. In our cohort, a mixed response to pembrolizumab was not uncommon $(12.9 \%)$. Despite the non-significant difference in the OS between the mixed and $\mathrm{CR}+\mathrm{PR}$ response groups, the OS of the mixed response group tended to be similar to that of the SD and PD response groups.

ICIs, anti-PD-L1 and anti-PD-1 antibodies have changed the treatment landscape for patients with advanced UC (18). In Japan, two ICIs, pembrolizumab and avelumab, have already been approved. Immunotherapeutics, even if they are made widely available to patients, bear a great challenge for clinicians as they have to accurately evaluate the clinical efficacy of these novel drugs in each patient. In particular, clinicians may face different response patterns of tumors treated with immunotherapeutics compared to the responses to conventional chemotherapeutic agents or other targeted therapies (19).

However, there have been few reports on mixed responses to immunotherapy, although they may bear another unconventional immune-related pattern, characterized by different responses across organs. These mixed reactions may be due to the heterogeneous clonality of tumor cells (20, 21). Recent studies have reported that the therapeutic effect of the ICI treatment varies from organ to organ in patients with non-small-cell lung cancer, hepatocellular cancer, melanoma and renal cell carcinoma $(15,22-24)$. However, only a few studies have examined the clinical association between a mixed response and the OS, performed only in patients with non-small-cell lung cancer and melanoma.

In a report of non-small-cell lung cancer in which 160 patients were treated with an anti-PD-1 or anti-PDL1 antibody, a mixed response was defined as 'dissociated responses', i.e., a concomitant decrease in certain tumoral elements and an increase in other elements, while the clinical benefit was defined as a minimum of six months of treatment with a beneficial effect on patients. Dissociated responses were observed in 12 patients $(7.5 \%), 6$ of who $(50 \%)$ showed a clinical benefit. In addition, existing adrenal lesions appear-ed to be more responsive to therapy. Patients with pseudoprogression or a dissociated response had a higher OS compared to those with a true progression (25).

In the report of metastatic melanoma, 292 patients were treated with anti-cytotoxic T-lymphocyte-associated protein 4 (anti-CTLA4), anti-PD1 and anti-CTLA4/anti-PD1 antibodies. Mixed responses were defined as simultaneously regressing and progressing metastatic lesions, based on the RECIST version 1.1 criteria. A mixed response was reported in 64 patients (22\%). Unsurprisingly, an OS analysis demonstrated the best outcomes in clinical responders and worst outcomes in clinical non-responders. The OS of the clinical mixed response group was intermediate (11).

We recently reported the organ-specific tumor response to pembrolizumab in advanced UC; however, we did not evaluate the clinical association between the mixed response and the OS. In this report, we evaluated the organ-specific therapeutic effect of pembrolizumab, and found a significant difference in the OS between patients with and without primary tumor organ disease and liver metastasis (17). A closer look at each of the four cases in the mixed response group revealed that three had a primary tumor organ disease and one had liver metastases. These patient backgrounds may have been responsible for the poor outcomes compared to previously reported findings $(11,25)$, wherein the OS in the mixed response group was better than that of the nonresponding group in cases of non-small-cell lung carcinoma and melanoma.

Several limitations associated with the present study warrant mention. This study was retrospective in nature. The absence of a statistically significant difference in OS between the mixed response group and $\mathrm{CR}+\mathrm{PR}$ group may have been due to the sample size; thus, our findings should be validated in larger prospective studies.

In conclusion, in this retrospective study we confirmed a mixed response in $12.9 \%$ of advanced UC patients who received pembrolizumab following failure of the platinumbased chemotherapy. While the mixed response group did not show a statistically significant difference in the OS compared to the $\mathrm{CR}+\mathrm{PR}$ response group, the value tended to be similar to that in the SD and PD response groups.

\section{Conflicts of Interest}

The Authors declare that they have no conflicts of interest for this study.

\section{Authors' Contributions}

NF, TN and MN designed the study. NF, NS, ST, FM, YS, YH and TT extracted the data. NS, FM, TT, NS and KK assisted with the data processing and statistical analysis. NF and $\mathrm{TN}$ wrote the article. NS, NS, KK and MN supervised the study and critically reviewed the manuscript. All Authors read and approved the final version of the manuscript.

\section{References}

1 Kim JW, Tomita Y, Trepel $\mathrm{J}$ and Apolo AB: Emerging immunotherapies for bladder cancer. Curr Opin Oncol 27(3): 191-200, 2015. PMID: 25811346. DOI: 10.1097/CCO. 0000000000000177 
2 Mariathasan S, Turley SJ, Nickles D, Castiglioni A, Yuen K, Wang Y, Kadel EE III, Koeppen H, Astarita JL, Cubas R, Jhunjhunwala S, Banchereau R, Yang Y, Guan Y, Chalouni C, Ziai J, Şenbabaoğlu Y, Santoro S, Sheinson D, Hung J, Giltnane JM, Pierce AA, Mesh K, Lianoglou S, Riegler J, Carano RAD, Eriksson P, Höglund M, Somarriba L, Halligan DL, van der Heijden MS, Loriot Y, Rosenberg JE, Fong L, Mellman I, Chen DS, Green M, Derleth C, Fine GD, Hegde PS, Bourgon R and Powles T: TGF $\beta$ attenuates tumour response to PD-L1 blockade by contributing to exclusion of T cells. Nature 554(7693): 544548, 2018. PMID: 29443960. DOI: 10.1038/nature25501

3 Bellmunt J, de Wit R, Vaughn DJ, Fradet Y, Lee JL, Fong L, Vogelzang NJ, Climent MA, Petrylak DP, Choueiri TK, Necchi A, Gerritsen W, Gurney H, Quinn DI, Culine S, Sternberg CN, Mai Y, Poehlein CH, Perini RF, Bajorin DF and KEYNOTE-045 Investigators: Pembrolizumab as second-line therapy for advanced urothelial carcinoma. N Engl J Med 376(11): 10151026, 2017. PMID: 28212060. DOI: 10.1056/NEJMoa1613683

4 Powles T, Park SH, Voog E, Caserta C, Valderrama BP, Gurney H, Kalofonos H, Radulović S, Demey W, Ullén A, Loriot Y, Sridhar SS, Tsuchiya N, Kopyltsov E, Sternberg CN, Bellmunt J, Aragon-Ching JB, Petrylak DP, Laliberte R, Wang J, Huang B, Davis C, Fowst C, Costa N, Blake-Haskins JA, di Pietro A and Grivas P: Avelumab maintenance therapy for advanced or metastatic urothelial carcinoma. N Engl J Med 383(13): 12181230, 2020. PMID: 32945632. DOI: 10.1056/NEJMoa2002788

5 Wang $\mathrm{Q}$, Gao $\mathrm{J}$ and $\mathrm{Wu} \mathrm{X}$ : Pseudoprogression and hyperprogression after checkpoint blockade. Int Immunopharmacol 58: 125-135, 2018. PMID: 29579717. DOI: 10.1016/j.intimp.2018.03.018

6 Han XJ, Alu A, Xiao YN, Wei YQ and Wei XW: Hyperprogression: A novel response pattern under immunotherapy. Clin Transl Med 10(5): e167, 2020. PMID: 32997401. DOI: $10.1002 / \mathrm{ctm} 2.167$

7 Hodi FS, Hwu WJ, Kefford R, Weber JS, Daud A, Hamid O, Patnaik A, Ribas A, Robert C, Gangadhar TC, Joshua AM, Hersey P, Dronca R, Joseph R, Hille D, Xue D, Li XN, Kang SP, Ebbinghaus S, Perrone A and Wolchok JD: Evaluation of immune-related response criteria and RECIST v1.1 in patients with advanced melanoma treated with pembrolizumab. J Clin Oncol 34(13): 1510-1517, 2016. PMID: 26951310. DOI: 10.1200/JCO.2015.64.0391

8 Kato S, Goodman A, Walavalkar V, Barkauskas DA, Sharabi A and Kurzrock R: Hyperprogressors after immunotherapy: Analysis of genomic alterations associated with accelerated growth rate. Clin Cancer Res 23(15): 4242-4250, 2017. PMID: 28351930. DOI: 10.1158/1078-0432.CCR-16-3133

9 Saâda-Bouzid E, Defaucheux C, Karabajakian A, Coloma VP, Servois V, Paoletti X, Even C, Fayette J, Guigay J, Loirat D, Peyrade F, Alt M, Gal J and Le Tourneau C: Hyperprogression during anti-PD-1/PD-L1 therapy in patients with recurrent and/or metastatic head and neck squamous cell carcinoma. Ann Oncol 28(7): 1605-1611, 2017. PMID: 28419181. DOI: 10.1093/ annonc/mdx 178

10 Ferrara R, Mezquita L, Texier M, Lahmar J, Audigier-Valette C, Tessonnier L, Mazieres J, Zalcman G, Brosseau S, Le Moulec S, Leroy L, Duchemann B, Lefebvre C, Veillon R, Westeel V, Koscielny S, Champiat S, Ferté C, Planchard D, Remon J, Boucher ME, Gazzah A, Adam J, Bria E, Tortora G, Soria JC, Besse B and Caramella C: Hyperprogressive disease in patients with advanced non-small cell lung cancer treated with PD-1/PDL1 inhibitors or with single-agent chemotherapy. JAMA Oncol 4(11): 1543-1552, 2018. PMID: 30193240. DOI: 10.1001/ jamaoncol.2018.3676

11 Rauwerdink DJW, Molina G, Frederick DT, Sharova T, van der Hage J, Cohen S and Boland GM: Mixed response to immunotherapy in patients with metastatic melanoma. Ann Surg Oncol 27(9): 3488-3497, 2020. PMID: 32472413. DOI: 10.1245/ s10434-020-08657-6

12 Sharma P and Allison JP: The future of immune checkpoint therapy. Science 348(6230): 56-61, 2015. PMID: 25838373. DOI: $10.1126 /$ science.aaa8172

13 Moch H, Cubilla AL, Humphrey PA, Reuter VE and Ulbright TM: The 2016 WHO Classification of tumours of the urinary system and male genital organs-part A: Renal, penile, and testicular tumours. Eur Urol 70(1): 93-105, 2016. PMID: 26935559. DOI: $10.1016 /$ j.eururo.2016.02.029

14 Eisenhauer EA, Therasse P, Bogaerts J, Schwartz LH, Sargent D, Ford R, Dancey J, Arbuck S, Gwyther S, Mooney M, Rubinstein L, Shankar L, Dodd L, Kaplan R, Lacombe D and Verweij J: New response evaluation criteria in solid tumours: revised RECIST guideline (version 1.1). Eur J Cancer 45(2): 228-247, 2009. PMID: 19097774. DOI: 10.1016/j.ejca. 2008.10.026

15 Schmid S, Diem S, Li Q, Krapf M, Flatz L, Leschka S, Desbiolles L, Klingbiel D, Jochum W and Früh M: Organspecific response to nivolumab in patients with non-small cell lung cancer (NSCLC). Cancer Immunol Immunother 67(12): 1825-1832, 2018. PMID: 30171269. DOI: 10.1007/s00262-0182239-4

16 Furubayashi N, Negishi T, Miura A, Nakamura N and Nakamura M: Organ-specific therapeutic effect of paclitaxel and carboplatin chemotherapy after platinum-based chemotherapy and pembrolizumab for metastatic urothelial carcinoma. Res Rep Urol 12: 455-461, 2020. PMID: 33117745. DOI: 10.2147/RRU. S270495

17 Furubayashi N, Negishi T, Sakamoto N, Shimokawa H, Morokuma F, Song Y, Hori Y, Tomoda T, Tokuda N, Seki N, Kuroiwa $\mathrm{K}$ and Nakamura M: Organ-specific tumor response to pembrolizumab in advanced urothelial carcinoma after platinumbased chemotherapy. Onco Targets Ther 14: 1981-1988, 2021. PMID: 33776447. DOI: 10.2147/OTT.S299724

18 Grisay G, Pierrard J, Confente C and Seront E: Future strategies involving immune checkpoint inhibitors in advanced urothelial carcinoma. Curr Treat Options Oncol 22(1): 7, 2020. PMID: 33269438. DOI: $10.1007 / \mathrm{s} 11864-020-00799-9$

19 Chiou VL and Burotto M: Pseudoprogression and immunerelated response in solid tumors. J Clin Oncol 33(31): 35413543, 2015. PMID: 26261262. DOI: 10.1200/JCO.2015.61.6870

20 Schmid K, Oehl N, Wrba F, Pirker R, Pirker C and Filipits M: EGFR/KRAS/BRAF mutations in primary lung adenocarcinomas and corresponding locoregional lymph node metastases. Clin Cancer Res 15(14): 4554-4560, 2009. PMID: 19584155. DOI: 10.1158/1078-0432.CCR-09-0089

21 Krantz D, Hartana CA, Winerdal ME, Johansson M, Alamdari F, Jakubczyk T, Huge Y, Aljabery F, Palmqvist K, Zirakzadeh AA, Holmström B, Riklund K, Sherif A and Winqvist O: Neoadjuvant chemotherapy reinforces antitumour $\mathrm{T}$ cell response in urothelial urinary bladder cancer. Eur Urol 74(6): 688-692, 2018. PMID: 30025882. DOI: 10.1016/j.eururo.2018.06.048 
22 Lu LC, Hsu C, Shao YY, Chao Y, Yen CJ, Shih IL, Hung YP, Chang CJ, Shen YC, Guo JC, Liu TH, Hsu CH and Cheng AL: Differential organ-specific tumor response to immune checkpoint inhibitors in hepatocellular carcinoma. Liver Cancer 8(6): 480-490, 2019. PMID: 31799205. DOI: 10.1159/ 000501275

23 Pires da Silva I, Lo S, Quek C, Gonzalez M, Carlino MS, Long GV and Menzies AM: Site-specific response patterns, pseudoprogression, and acquired resistance in patients with melanoma treated with ipilimumab combined with anti-PD-1 therapy. Cancer 126(1): 86-97, 2020. PMID: 31584722. DOI: $10.1002 /$ cncr.32522

24 Negishi T, Furubayashi N, Nakagawa T, Nishiyama N, Kitamura H, Hori Y, Kuroiwa K, Son Y, Seki N, Tomoda T, Okajima E and Nakamura M: Site-specific response to nivolumab in renal cell carcinoma. Anticancer Res 41(3): 1539-1545, 2021. PMID: 33788747. DOI: 10.21873/anticanres.14913
25 Tazdait M, Mezquita L, Lahmar J, Ferrara R, Bidault F, Ammari S, Balleyguier C, Planchard D, Gazzah A, Soria JC, Marabelle A, Besse B and Caramella C: Patterns of responses in metastatic NSCLC during PD-1 or PDL-1 inhibitor therapy: Comparison of RECIST 1.1, irRECIST and iRECIST criteria. Eur J Cancer 88: 38-47, 2018. PMID: 29182990. DOI: 10.1016/j.ejca. 2017.10.017
Received May 25, 2021

Revised June 21, 2021

Accepted July 2, 2021 